\title{
Fecha de aplicación de fuego y diversidad de herbáceas en una sabana de Elionorus muticus (Spreng) O. Kuntze
}

\author{
Date of prescribed fire and herbaceous diversity of an Elionorus muticus (Spreng.) \\ O. Kuntze savanna
}

\begin{abstract}
CARLOS KUNST ${ }^{1}$ SANDRA BRAVO ${ }^{2}$, FABIO MOSCOVICH ${ }^{1}$, JULIO HERRERA $^{1}$, JOSÉ GODOY ${ }^{1} \&$ SILVIA VÉLEZ ${ }^{3}$

${ }^{1}$ Instituto Nacional de Tecnología Agropecuaria, Estación Experimental Agropecuaria Santiago del Estero, Jujuy 850, 4200, Santiago del Estero, Argentina; e-mail: ckunst@ correo.inta.gov.ar

${ }^{2}$ Universidad Nacional de Santiago del Estero, Facultad de Ciencias Forestales, Cátedra de Botánica General, Belgrano (S) 1912, 4200, Santiago del Estero, Argentina

${ }^{3}$ Universidad Nacional de Santiago del Estero, Facultad de Ciencias Forestales, Instituto de Tecnología de la Madera, Laboratorio de Energía de Biomasa, Belgrano (S) 1912, 4200, Santiago del Estero, Argentina
\end{abstract}

\begin{abstract}
RESUMEN
El fuego es a la vez una perturbación natural y una herramienta agropecuaria en las sabanas de Elionorus muticus (Spreng) O. Kuntze de la región chaqueña argentina. Nuestro objetivo fue evaluar el efecto de la fecha de aplicación de fuego sobre la diversidad de herbáceas de la sabana. Para ello se quemaron parcelas en cuatro fechas sucesivas, desde fines de julio hasta principios de octubre, en dos experimentos iniciados en 1993 y 1994, respectivamente. La riqueza y abundancia de especies de gramíneas y latifoliadas se registraron antes del fuego; y después del mismo, al final de las tres estaciones de crecimiento siguientes. La información fue resumida en el índice de diversidad de ShannonWiener. Para el análisis estadístico se calculó $\Delta \mathrm{H}^{\prime}$, definida como la diferencia entre los índices H' antes y después del fuego. Esta variable fue sometida a un análisis de varianza, empleando la fecha de fuego, experimento, su interacción y año de observación como variables independientes, con un enfoque de medidas repetidas y en dos escalas de tiempo: a corto y largo plazo. Los resultados indican que el fuego, en general, produce un aumento de la diversidad de herbáceas. La fecha de aplicación del fuego modifica ese resultado de manera significativa, hecho que se atribuye a las condiciones ambientales de pre y post-quema específicas de cada fecha y al estado fenológico de las especies. Las fechas de fuego que causan un mayor incremento en la diversidad son agosto-septiembre, a mediados de la temporada de fuego, hecho que se atribuye a una mayor resistencia de las plantas al fuego y a las mejores condiciones climáticas post-fuego.
\end{abstract}

Palabras clave: sabana, fuego, diversidad herbáceas, región chaqueña.

\begin{abstract}
Fire is both a natural disturbance and a range management tool in the savannas of Elionorus muticus (Spreng) O. Kuntze, of the Chaco region of Argentina. Our objective was to assess the effect of different dates of fire application on the herbaceous diversity of the savanna. Plots were burn at random in four successive dates, in two experiments initiated in 1993 and 1994, respectively. Richness and abundance of grasses and broadleaf species were registered in each plot, before the fire and after three growing seasons from fire. Data were summarized using the Shannon-Wiener diversity index $\left(\mathrm{H}^{\prime}\right)$. A variable $\Delta \mathrm{H}^{\prime}$ was defined as the difference between the $\mathrm{H}^{\prime}$ observed before and after the fire for each plot/ year, and used as dependent variable in an ANOVA; fire application date, experiment, their interaction and year of observation $(\mathrm{t})$ were considered as independent variables, with a repeated measures approach. Results indicate that although fire increased herbaceous diversity significantly, this general outcome was significantly modified by the date of fire application. This result is attributed to the pre and post fire environmental conditions as well as the phenological status of the species. Fire application at the mid-fire season generates higher diversity than any other time, probably due to plant resistance and better post fire environmental conditions.
\end{abstract}

Key words: savanna, fire, herbaceous diversity, Chaco region. 


\section{INTRODUCCIÓN}

La vegetación chaqueña está compuesta por un mosaico de comunidades leñosas (bosques y 'arbustales'1) y herbáceas (sabanas de aibe y pastizales, Bucher 1982). En las sabanas, la gramínea Elionorus muticus (Spreng.) O. Kuntze (aibe, espartillo o paja amarga) es generalmente la especie dominante, alcanzando valores entre 30 y $90 \%$ de cobertura (Cabrera \& Willink 1973, Kunst et al. 1985). En sabanas no degradadas por sobrepastoreo y/o excesiva frecuencia de fuego pueden encontrarse también abundantes ejemplares de Pennisetum frutescens, Schyzachirium tenerum, Heteropogon contortus, Pappophorum pappipherum, Rynchosia senna, Indigofera parodiana, Zexmenia spp entre otras gramíneas y latifoliadas herbáceas (Renolfi 1993, Kunst et al. 1998, Radrizzani et al. $2001^{2}$ ). Es común también la presencia de arbustos de los géneros Acacia, Prosopis, Celtis y Schinus; y subarbustos del género Baccharis (C. Kunst observación personal).

Las matas de aibe son cespitosas, con rizoma brevísimo y de forma semicircular, de $40-50 \mathrm{~cm}$ de altura. Poseen una gran densidad de hojas finas y alargadas, que contienen terpeno citral, característica que les otorga gran inflamabilidad (Burkart 1969) y que junto a las características climáticas de la región, transforma a las sabanas en un ambiente propenso al fuego.

El fuego es una perturbación frecuente en el paisaje natural chaqueño. Antiguamente los indígenas usaban el fuego para la caza y la guerra (Bucher 1982). Dentro de las comunidades de leñosas se considera el fuego como una perturbación intensa (Tálamo 1999) y es el factor responsable del mantenimiento de las sabanas como comunidades 'abiertas', dominadas por herbáceas (Morello \& Adamoli 1974, Bucher 1982). En la actualidad, los ganaderos queman las sabanas de aibe a fin de obtener el rebrote verde de los pastos y controlar leñosas (Morello \& Saravia Toledo 1959).

${ }^{1}$ Voz local que describe comunidades vegetales compuesta por arbustos pertenecientes a los géneros Acacia, Celtis y Schinus, altura 1-3 m.

${ }^{2}$ RADRIZZANI A, C KUNST \& R RENOLFI (2001) Aplicación del modelo de estados y transiciones al manejo de pastizales de Elionorus muticus en el Chaco santiagueño. Asociación Argentina de Manejo de Pastizales Naturales, Memorias del Primer Congreso Nacional, San Cristóbal, Santa Fé, Argentina: 50-51.
La ausencia prolongada de fuego en aibales produce una acumulación excesiva de biomasa aérea de la especie dominante, lo que influye negativamente sobre la diversidad de herbáceas acompañantes (C. Kunst observación personal). Existe evidencia de que algunas especies, tales como Heteropogon contortus, integrante natural del aibal, necesitan de la recurrencia del fuego para mantenerse en el tiempo (Tothill 1969, Orr et al. 1997).

Las sabanas de aibe tienen gran importancia ecológica ya que son hábitat de numerosas especies vegetales y animales nativas. Desde el punto de vista económico, son una fuente de forraje para la ganadería bovina extensiva (Kunst et al. 1985, 1998, Radrizzani et al. 2001²). Por lo tanto, su conservación es un objetivo de interés ecológico y agronómico. La predisposición de la sabana al fuego hace necesario el estudio de prácticas racionales y apropiadas de quemas con objetivos de manejo.

Debido a las características climáticas de la región chaqueña, los fuegos en las sabanas ocurren desde principios de junio, cuando comienza la estación seca y las primeras heladas matan la parte aérea de la vegetación herbácea; hasta fines de octubre, cuando las lluvias y altas temperaturas generan el nuevo ciclo anual de crecimiento. Aunque la frecuencia natural en los aibales sería un fuego cada 2-4 años, en áreas sujetas a pastoreo se lo emplea anualmente (Bordón 1993, Bravo et al. 2001).

Existen protocolos técnicos desarrollados en regiones con características similares a la chaqueña que permiten el manejo del fuego para controlar especies leñosas (Britton et al. 1987, Trollope 1984) y que han sido adaptados a estos ambientes con idénticos objetivos (Kunst et al. 2000). Dentro de esas normas, el efecto de distintas épocas de aplicación de fuego sobre la diversidad herbácea de la sabana es un aspecto aún no estudiado.

La diversidad de las comunidades bióticas es un atributo específico, relacionado con su identidad y presencia en el ecosistema (Begon et al. 1997) y sus variaciones pueden utilizarse como indicadores del impacto de perturbaciones naturales y antrópicas (Clarke \& Warwick 1994, Jorgensen \& Padisák 1996, Tálamo 1999).

En otros ecosistemas de pastizales del mundo, la aplicación de fuegos en distintas épocas del año, a intervalos diferentes de tiempo, ejercen impactos diferenciales sobre la composición botánica de las herbáceas (Silva 1987, Hansmire et al. 1988, Howe 1994, 1995, Gill \& McCarthy 1998) A lo largo de la extensa temporada de fuego en la región chaqueña, las plantas de la sabana 
atraviesan distintas fases fenológicas, por lo cual es lógico esperar una respuesta diferencial frente a la misma perturbación. Es necesario tener en cuenta también el ambiente pre y postquema.

El objetivo de este trabajo fue evaluar el impacto de distintas fechas de aplicación de fuego sobre la diversidad de herbáceas en sabanas de aibe. Esta información resulta necesaria para analizar la factibilidad de aplicación del fuego, valorar sus consecuencia y generar recomendaciones para su uso como herramienta de manejo.

\section{MATERIALES Y MÉTODOS}

\section{Sitio de estudio}

El trabajo se desarrolló en el Campo Experimental 'La María', perteneciente a la Estación Experimental Agropecuaria Santiago del Estero, Instituto Nacional de Tecnología Agropecuaria, Santiago del Estero, Argentina (28 $\left.03^{\circ} \mathrm{S}, 6^{\circ} 15^{\prime} \mathrm{O}\right)$. El clima es semiárido subtropical, con una precipitación promedio anual de $574 \mathrm{~mm}$ (extremos 260$1.072 \mathrm{~mm}$, período 1934-2000, Observatorio EEA Santiago del Estero, INTA), inviernos fríos y secos, y veranos calientes y lluviosos (Boletta 1988).

El material experimental fue una sabana dominada por aibe ubicada en el extremo oeste del Campo Experimental. El suelo de la sabana fue caracterizado como un Torriorthentic Haplustol, limo-arenoso, mixto, hipertérmico (Lorenz 1995 comunicación personal). Los sectores utilizados para los experimentos no habían sido quemados en los últimos 5 años.

\section{Experimentos de campo}

Se realizaron dos ensayos, que fueron iniciados en 1993 y 1994 (EXP1 y EXP2 respectivamente). Las unidades experimentales fueron parcelas de 15 x 15 m. Los sectores tratados solo estuvieron abiertos a la fauna silvestre.

En forma previa al fuego y en cada parcela se estimó: (a) presencia y número de individuos de especies herbáceas (gramíneas y latifoliadas). Para ello se instalaron dos transectas fijas de $10 \mathrm{~m} \mathrm{de}$ largo. En cada metro se localizó un marco de 0,1 $\mathrm{m}^{2}$, en forma paralela al sentido de la transecta, registrándose los datos mencionados (Anderson 1980, Bonham 1989); (b) carga de combustible fino. Se midió sólo el estrato herbáceo por ser el que influye en mayor medida sobre el comportamiento del fuego en la sabana (Rothermel 1983). El combustible fino se dividió en biomasa aérea en pié y mantillo. El primero se estimó mediante el método de corte en cinco cuadrantes de $50 \mathrm{~cm}$ x 50, distribuídos al azar. El mantillo se recolectó a mano en cada cuadrante. Las muestras de biomasa aérea y mantillo se pesaron en el campo y se secaron en estufa a $60^{\circ} \mathrm{C}$ para determinar el contenido de humedad. Los resultados se expresaron en $\mathrm{kg} \mathrm{ha}^{-1}$ (materia seca) y en porcentaje de agua; (c) contenido de humedad del suelo (\%), se determinó en los primeros $15 \mathrm{~cm}$ utilizando gravimetría; (d) datos climáticos, se midieron durante la quema la temperatura $\left({ }^{\circ} \mathrm{C}\right)$ y la humedad relativa del aire (\%) a $1,5 \mathrm{~m}$ de altura del suelo, con aparatos meteorológicos de campaña.

Para la caracterización de cada fecha de quema se tuvieron en cuenta además la temperatura $\left({ }^{\circ} \mathrm{C}\right)$ del aire a $50 \mathrm{~cm}$ y a $5 \mathrm{~cm}$ de altura sobre el nivel del suelo y otros datos climáticos, entre ellos heladas agronómicas (temperaturas $<10^{\circ} \mathrm{C}$ ), y fecha de lluvias, correspondientes a la semana previa a la aplicación del fuego y que fueron obtenidos de la estación meteorológica ubicada en el Campo Experimental. Se tuvieron en cuenta las temperaturas citadas ya que las especies de gramíneas nativas presentan síndrome fotosintético $\mathrm{C}_{4}$ y resultan por lo tanto afectadas por temperaturas inferiores a $10^{\circ} \mathrm{C}$ (Larcher 1995)

En condiciones consideradas propicias según los protocolos de referencia (Britton et al. 1987), se les aplicó fuego a dos parcelas seleccionadas al azar de manera consecutiva entre julio y octubre cada 15 días aproximadamente. Se empleó un fuego frontal en forma perpendicular al viento dominante. Este tipo de fuego predomina en las sabanas (Bravo et al. 2001). Se utilizaron antorchas de goteo para la ignición. Las características de los fuegos fueron descriptas de acuerdo a lo propuesto por Alexander (1982) y Ryan \& Noste (1985).

El efecto del fuego sobre el estrato herbáceo se evaluó mediante lectura de las dos transectas instaladas en cada parcela al final de las tres estaciones de crecimiento siguientes a su aplicación, en forma similar a lo expuesto en el párrafo anterior. En el EXP1 también se registraron datos en Enero de 1994 a fin de evaluar cambios de corto plazo. Se realizó esta medición, ubicada en la mitad del período de crecimiento, para poder detectar la presencia de herbáceas de ciclo anual o bianual que desaparecen rápidamente debido a la dominancia del aibe (Kunst observación personal).

\section{Procesamiento de datos}

Los efectos de perturbaciones en comunidades pueden analizarse mediante dos enfoques (Clarke \& Warwick 1994): análisis multivariado (e.g., 
componentes principales); y síntesis de toda la información en un índice y posterior análisis estadístico del mismo. En este estudio se utilizó este último enfoque.

La diversidad de especies se estimó para cada parcela por el índice de Shannon-Wiener (H', Ludwig \& Reynolds 1988):

$$
\mathrm{H}^{\prime}=-\Sigma[\mathrm{Pi} *(\ln \mathrm{Pi})],
$$

donde Pi es el porcentaje de individuos de una especie sobre el total de individuos en la parcela y ln el logaritmo en base e. Para el cálculo de H' se empleó el paquete estadístico PRIMER (Carr 1996).

Se graficó el índice H' en función de los años de muestreo a fin de observar su dinámica a través del tiempo. Se consideró a H' como una variable continua.

\section{Análisis estadístico}

Las fechas de aplicación del fuego (EF) se ordenaron en función de sus características climáticas de pre y post-quema en cuatro épocas sucesivas, numeradas de 1 a 4 (Tabla 1) y se consideraron como factores de clasificación (Cox 1958). Se tuvieron en cuenta dos períodos de tiempo postfuego: corto (hasta 6 meses) y largo plazo (primera a tercera estación de crecimiento).

Para la evaluación a corto plazo se utilizaron los H' obtenidos en forma previa al fuego durante 1993 y los correspondientes al mes de enero 1994. Se calculó dicha diferencia para cada parcela en todas las fechas de aplicación de fuego, obteniéndose la variable $\Delta \mathrm{H}^{\prime}$ :

$$
\Delta \mathrm{H}^{\prime}=(-1) *\left(\mathrm{H}^{\prime} \text { antes }-\mathrm{H}^{\prime} \text { después }\right)
$$

Conceptualmente, la variable $\Delta \mathrm{H}^{\prime}$ representa los cambios generados en la diversidad de especies herbáceas por efecto del fuego. Una magnitud de $\Delta \mathrm{H}^{\prime}>0$ se interpretó como un aumento en la diversidad a causa del fuego; y viceversa si $\Delta H^{\prime}$ es igual o menor que cero. Esta variable se empleó como dependiente en un análisis de varianza con EF como variable independiente. La variable EF representa el efecto del conjunto de circunstancias ambientales y fenológicas características de cada fecha de aplicación de fuego.

Para el período de largo plazo se empleó el mismo concepto, pero utilizando datos de EXP1 y EXP2. Se usaron los índices H' observados antes del fuego en cada parcela y los H' calculados al final de cada estación de crecimiento para cada experimento, hasta tres años post-quema, respectivamente, de acuerdo a la siguiente fórmula:

$$
\Delta H^{\prime} t=(-1)^{*}\left(H^{\prime} \text { antes del fuego }-H^{\prime} t\right),
$$

donde $\mathrm{H}^{\prime}$ antes del fuego = índice H' observados en 1993 para EXP1 y 1994 para EXP2; y H't = índice H' observado en $\mathrm{t}=1994,1995$ y 1996 para EXP1; y t $=1995,1996$ y 1997 para EXP2, respectivamente y para parcela. El período ' $t$ ' se refiere al año de observación o número de estación de crecimiento luego del fuego. $\Delta \mathrm{H}^{\prime} \mathrm{t}$ fue interpretada de la misma manera que $\Delta H^{\prime}$.

TABLA 1

Algunas características ambientales de épocas de fuego, experimentos de 1993 y 1994. Sabana de Elionorus muticus (Spreng.) O. Kuntze, Campo Experimental 'La María', INTA EEA Santiago del Estero

\begin{tabular}{|c|c|c|c|c|c|c|c|}
\hline \multirow[t]{2}{*}{$\begin{array}{l}\text { Año } \\
(\mathrm{EXP})\end{array}$} & \multirow[t]{2}{*}{$\begin{array}{c}\text { Fecha de quema } \\
\text { (EF) }\end{array}$} & \multirow[t]{2}{*}{$\begin{array}{l}\text { Fecha } \\
\text { primera lluvia } \\
>10 \mathrm{~mm}\end{array}$} & \multirow[t]{2}{*}{$\begin{array}{l}\text { Número de } \\
\text { días hasta } \\
\text { primera lluvia }\end{array}$} & \multicolumn{2}{|c|}{$\begin{array}{c}\text { Temperatura media } \\
\text { del aire }\left({ }^{\circ} \mathrm{C}\right) \\
\text { período (Observatorio) }\end{array}$} & \multirow{2}{*}{$\begin{array}{l}\text { Temperatura de aire }\left({ }^{\circ} \mathrm{C}\right) \\
\text { (momento de quema, } \\
\text { altura } 1,5 \mathrm{~m} \text { ) } \\
\text { na }\end{array}$} & \multirow[t]{2}{*}{$\begin{array}{l}\text { Número heladas } \\
\text { agronómicas } \\
\left(\mathrm{T}<10^{\circ} \mathrm{C}\right)\end{array}$} \\
\hline & & & & Media máxima & Media mínima & & \\
\hline \multirow[t]{5}{*}{1993} & 15/7 (EF 1) & $31 / 10$ & 108 & 25,2 & 5,6 & 16 & 31 \\
\hline & $13 / 8$ (EF 2) & & 79 & 25.3 & 7,2 & 15,6 & 12 \\
\hline & 22/8 (EF 2) & & 70 & 26,7 & 9,1 & 24,5 & 7 \\
\hline & 16/9 (EF 3) & & 45 & 26,1 & 12,4 & 28,5 & 0 \\
\hline & $6 / 10(\mathrm{EF} 4)$ & & 25 & 29,9 & 13,1 & 26,7 & 0 \\
\hline \multirow[t]{4}{*}{1994} & $13 / 7$ (EF 1) & $23 / 10$ & 102 & 26,3 & 9,8 & 26,7 & 21 \\
\hline & $3 / 8$ (EF 2) & & 76 & 27,3 & 11,2 & 16 & 15 \\
\hline & $5 / 9$ (EF 3) & & 48 & 29,5 & 14,4 & 25 & 2 \\
\hline & $23 / 9(\mathrm{EF} \mathrm{4})$ & & 30 & 29,4 & 16,2 & 34 & 0 \\
\hline
\end{tabular}

Some environmental features of fire application dates, experiments of 1993 and 1994. Savanna of Elionorus muticus (Spreng.) O. Kuntze, 'La María’ Experimental Ranch, INTA EEA Santiago del Estero 
En el análisis de varianza de la variable dependiente $\Delta \mathrm{H}^{\prime} \mathrm{t}$ los factores de clasificación fueron experimento (EXP), EF, año de observación (t) y sus respectivas interacciones. Se utilizó un enfoque de medidas repetidas en el tiempo (Kirkman et al. 1998, Littel et al. 1998). La variable EXP representa el efecto combinado del cambio de sitio experimental y de las circunstancias climáticas específicas del año de comienzo. La interpretación de EF fue desarrollada en el párrafo anterior. Año de observación o período (t) representa los efectos combinados de la dinámica propia de la vegetación y del clima.

El índice de correlación de Pearson $(\varphi)$ se empleó para estudiar la asociación entre variables. Se removió el efecto de las diferencias intrínsecas de $H^{\prime}$ entre parcelas al utilizar $\Delta H^{\prime}$ y $\Delta H^{\prime} t$ como variables dependientes. Debido a la heterogeneidad de la varianzas de $\Delta \mathrm{H}^{\prime}$, la misma fue transformada a rangos (Conover 1980).

Para los cálculos estadísticos del período de corto plazo se emplearon los procedimientos MEANS, GLM y CORR del paquete estadístico SAS (Freund et al. 1986, SAS 1990, Littell et al. 1998). Para el largo plazo se empleó el procedimiento $5 \mathrm{~V}$ del paquete estadístico BMDP (Dixon 1992), que utiliza la prueba de Wald para evaluar el grado de significancia estadística de las variables independientes. Las variables independientes no significativas estadísticamente fueron incorporadas al error experimental (Underwood 1997). Para el cálculo de medias se utilizó el procedimiento LSMEANS y para su comparación el test de t (SAS 1990). Se empleó la suma de cuadrados tipo III para las pruebas de F. En todos los análisis estadísticos se empleo un $\alpha=0,1$.

\section{RESULTADOS Y DISCUSIÓN}

La cantidad de combustible fino (promedio general) en todas las parcelas fue $10.000 \mathrm{~kg} \mathrm{ha}^{-1}$, correspondiendo $6.000 \mathrm{~kg} \mathrm{ha}^{-1}$ a la biomasa en pie y $4.000 \mathrm{~kg} \mathrm{ha}^{-1}$ al mantillo (peso verde). El contenido de humedad del combustible se estimó entre 16-20\% para la biomasa aérea en pie, mientras que en el mantillo osciló entre un 10-15\%. La biomasa en pié fue el principal componente de la fase de combustión activa del frente de fuego, mientras que el centro de las matas de pasto y el mantillo continuaron ardiendo luego del pasaje del frente principal, constituyendo una fase de combustión secundaria. El largo promedio de las llamas correspondiente a la fase de combustión activa osciló entre 1,6 y 3,5 m, lo que implica una intensidad frontal entre 760 y $3.900 \mathrm{~kW} \mathrm{~m}^{-1}$, sin tener en cuenta la fase de combustión secundaria y los fuegos de rescoldo. El consumo de combustible fino fue total. La ceniza fue de color negro y el suelo de las parcelas tratadas no cambió de color. En los dos experimentos (EXP1 y EXP2) se observó, en el mes de diciembre siguiente al fuego, abundante material verde, producto de rebrotes de plantas sobrevivientes y del establecimiento de nuevas plántulas.

Los fuegos obtenidos fueron clasificados como de alta intensidad, debido al largo de las llamas y de severidad media a baja por el grado de consumo de combustible, especialmente del mantillo.

Los ambientes pre y post-quema de los fuegos tempranos fueron diferentes de los encendidos tardíamente debido a los siguientes aspectos: (a) el contenido de agua del suelo disminuyó y la temperatura del aire a $50 \mathrm{~cm}$ y $5 \mathrm{~cm}$ sobre el nivel del suelo aumentó a medida que avanzó la temporada de fuego (Fig. 1); (b) en EF 1 y EF 2 la temperatura del aire en los dos niveles menciona-
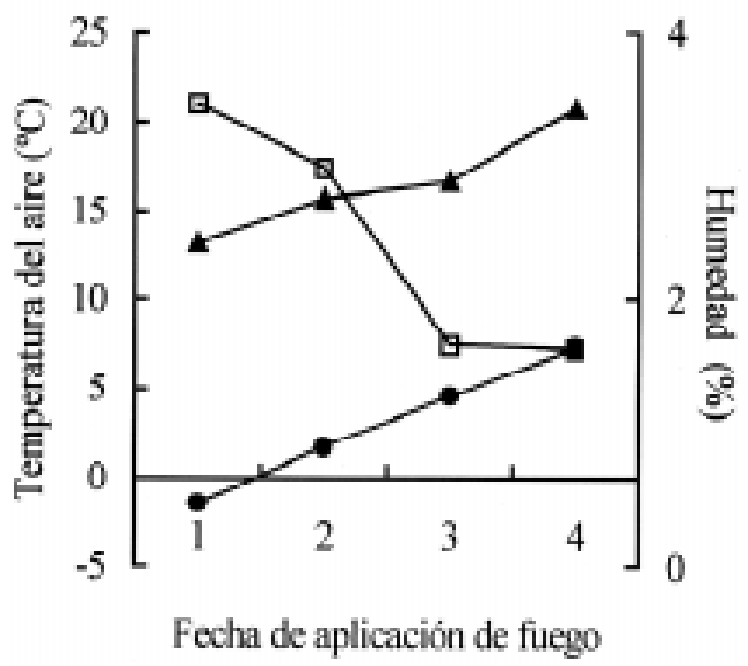

Fig. 1: Condiciones ambientales observadas en las cuatro épocas de aplicación de fuego: temperatura del aire $\left({ }^{\circ} \mathrm{C}\right)$ registradas a $0,5 \mathrm{~m}(\bullet)$ y $0,05 \mathrm{~m}(\boldsymbol{\Delta})$ sobre el suelo; y contenido de humedad del suelo (\%), profundidad 0-15 $\mathrm{cm}(\square)$. Temperaturas observadas en Observatorio Meteorológico, Campo Experimental 'La María', INTA EEA Santiago del Estero, promedio 5 días previos a quemas. Contenido de humedad observado en parcelas, inmediatamente antes fuego. Promedios de los dos experimentos (1993 y 1994).

Environmental conditions observed at the four fire application dates at fire: air temperatures $\left({ }^{\circ} \mathrm{C}\right)$ registered at $0.5 \mathrm{~m}(\mathbf{O})$ and $0.05 \mathrm{~m} \mathrm{( \Delta )}$ above soil surface; and soil water content (\%), 0-15 cm depth $(\square)$. Air temperatures observed at the Weather Station, 'La María' Experimental Ranch, INTA EEA Santiago del Estero, average 5 days prefire. Soil water content observed at plots, immediately before fire. Means of both experiments (1993 and 1994). 
dos fue baja y el contenido de agua del suelo fue elevado en relación al observado en forma previa al fuego. Sin embargo, las plantas debieron esperar casi 3 meses hasta encontrar nuevamente condiciones térmicas favorables para el crecimiento y estuvieron sometidas a numerosas heladas (Tabla 1, Fig. 1); (c) en EF 3 y EF 4 a las dos alturas analizadas, la temperatura del aire fue alta y el contenido de agua del suelo fue menor al existente antes de la aplicación del fuego (Fig. 1, Tabla 1). Las condiciones ambientales post-quema fueron mejores que en las EF1 y EF2: las plantas no sufrieron temperaturas bajo cero y pudieron rebrotar y cubrir el suelo rápidamente después de caídas las primeras precipitaciones.

El estado fenológico de las especies varió a lo largo de la temporada de fuego. En julio-agosto estaban en receso mientras que en octubre ya presentaban algunos rebrotes verdes.

El índice H' observado en las parcelas antes del fuego presentó variabilidad, con extremos entre 0,71 y 1,93 . Ello se atribuye a que la distribución de las especies herbáceas dentro de la sabana estudiada no es al azar. Aunque el aibe es la especie dominante y se distribuye casi uniformemente, las especies acompañantes se disponen en un patrón agregado. Esta característica es propia de sabanas y pastizales de distintas regiones del mundo y se relaciona con la presencia de microrelieves que afectan la escorrentía y distribución del agua en el suelo (Burke et al. 1999). Las parcelas empleadas en el EXP1 presentaron mayor diversidad inicial que las utilizadas en el EXP2 (0,98-1,93 versus 0,38-1,03).

\section{Efectos a corto plazo}

La diversidad de especies, H' aumentó luego del fuego; y la media general de $\Delta \mathrm{H}^{\prime}$ fue significativamente mayor que cero (Tabla 2). El efecto de EF sobre $\Delta \mathrm{H}^{\prime}$ fue estadísticamente significativo $(\mathrm{P}<0,04)$. Las EF 3 y EF 4 se diferenciaron en forma significativa de las EF 1 y EF 2 en su magnitud promedio de $\Delta \mathrm{H}^{\prime}$ (Tabla 2 ). Se observó una correlación positiva $(\mathrm{t}$ de Pearson $=$ $0,70)$ y significativa $(\mathrm{P}<0,02)$ entre los índices $\mathrm{H}^{\prime}$ calculados antes y después del fuego, cuando se consideran en conjunto los datos de EXP y EF.

\section{Efectos a largo plazo}

Luego del fuego el índice H' aumentó en todas las fechas de aplicación en los dos EXP (Fig. 2A y $3 \mathrm{~A})$. La media por período de $\Delta \mathrm{H}$ 't fue significativamente mayor que cero en $\mathrm{t}=1$, pero su magnitud fue disminuyendo con el tiempo, y en $\mathrm{t}=3$ no difirió significativamente de cero (Fig. $2 \mathrm{~B}$ y $3 \mathrm{~B}$, Tabla 2).

Los efectos de EXP y EF sobre $\Delta \mathrm{H}^{\prime}$ t fueron altamente significativos $(\mathrm{P}<0,000$ y $\mathrm{P}<0,0008$, respectivamente). El efecto de EXP se atribuye principalmente a diferencias en la composición botánica inicial entre los sitios experimentales, ya discutida. El mayor aumento promedio en $\mathrm{H}^{\prime}$

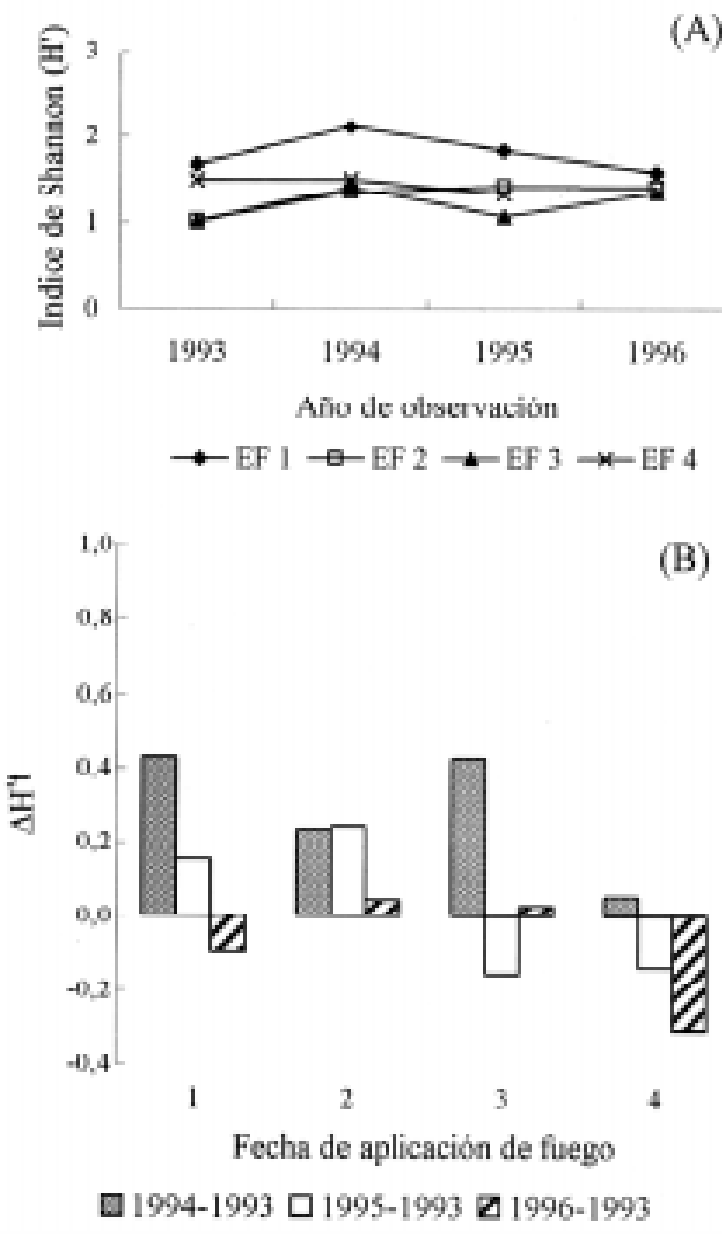

Fig. 2: Dinámica a través del tiempo de: (A) índice de diversidad de Shannon-Wiener (H'); y (B) diferencia entre H' observados antes y despúes del fuego $\left(\Delta H^{\prime} t\right)$, para cuatro épocas de quema de una sabana de Elionorus muticus (Spreng) O.

Kuntze. Ensayo iniciado en 1993 (EXP 1). Campo Experimental 'La María', EEA Santiago del Estero. EF: Epocas de fuego. Para otros datos ver Tabla 1 y texto.

Temporal dynamics of: (A) Shannon-Wiener diversity index $\left(\mathrm{H}^{\prime}\right)$; and (B) difference of H' observed before and after the fire $\left(\Delta \mathrm{H}^{\prime} \mathrm{t}\right)$, in four fire application dates in a Elionorus muticus savanna (Spreng.) O. Kuntze. Experiment initiated in 1993 (EXP 1). 'La María' Experimental Ranch, INTA EEA Sgo del Estero. EF: Fire application dates. For other information see Table 1 and text. 
TABLA 2

Impacto de épocas de aplicación de fuego (EF) sobre el promedio de $\Delta \mathrm{H}^{\prime}$ en el corto y largo plazo, considerando EXP 1 y EXP 2. Sabana de Elionorus muticus (Spreng) O. Kuntze (aibe), Campo Experimental 'La María', EEA Santiago del Estero

Effect of fire application dates on mean $\Delta \mathrm{H}^{\prime}$ in the short and long time span, considering EXP 1 and EXP 2. Savanna of Elionorus muticus (Spreng.) O. Kuntze, 'La María' Experimental Ranch, INTA EEA Santiago del Estero

(A) Corto plazo

EF $\quad \Delta H^{\prime}$ media $^{1}$

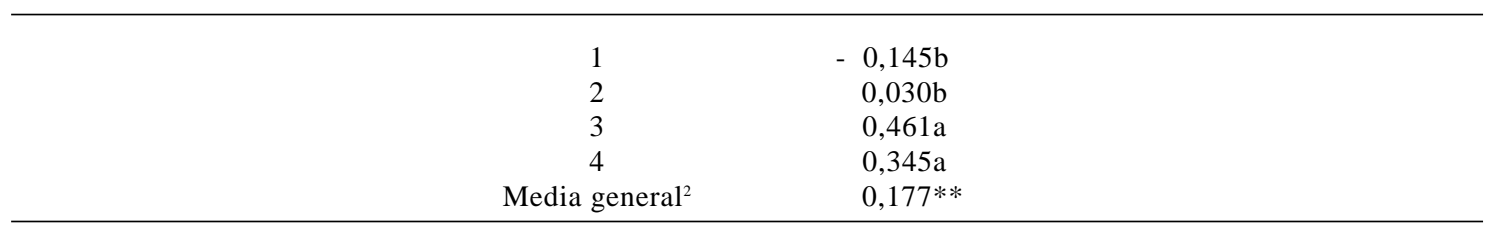

(B) Largo plazo

Estaciones de crecimiento después del fuego ${ }^{1} \quad$ Media general por EF $^{1}$

\begin{tabular}{ccccc}
\hline & 1 & 2 & 3 & $\Delta \mathrm{H}^{\prime} \mathrm{t}$ \\
$\mathrm{EF}$ & $\Delta \mathrm{H}^{\prime}$ & $\Delta \mathrm{H}^{\prime}$ & $\Delta \mathrm{H}^{\prime}$ & $0,265 \mathrm{a}$ \\
2 & $0,281 \mathrm{~b}$ & $-0,009 \mathrm{a}$ & $0,101 \mathrm{a}$ & $0,410 \mathrm{a}$ \\
3 & $0,357 \mathrm{a}, \mathrm{b}$ & $0,244 \mathrm{a}$ & $0,233 \mathrm{a}$ & $0,353 \mathrm{a}$ \\
4 & $0,749 \mathrm{a}$ & $0,381 \mathrm{a}$ & $0,446 \mathrm{a}$ & $-0,040 \mathrm{~b}$ \\
Media general $^{2}$ & $0,353 \mathrm{a}, \mathrm{b}$ & $0,024 \mathrm{a}$ & $0,101 \mathrm{a}$ & 0,120 \\
\hline
\end{tabular}

${ }^{1}$ Medias de cada estación de crecimiento estimadas mediante procedimiento LSMEANS (SAS 1988). En cada columna, cifras seguidas de letras diferentes indican diferencias significativas, $\alpha=0,10$, prueba t de Student

${ }^{2}$ Promedio estimado mediante método tradicional

** Significativamente diferente de cero $(\mathrm{P}<0,05)$

se presenta en el EXP2, en el cual la diversidad inicial es menor.

La EF 2 presentó el mayor promedio general de $\Delta \mathrm{H}^{\prime} \mathrm{t}$, con una magnitud siempre mayor que cero. Sólo la EF 4 presentó magnitudes bajas (Fig. 2B y 3B, Tabla 2).

La correlación entre el H' inicial y los H' observados luego del fuego fue significativa en las dos primeras estaciones de crecimiento luego del fuego $(\rho$ de Pearson $=0,74 ; \mathrm{P}<0,0007$ y $\rho=0,66 ; \mathrm{P}$ $<0,0051$, respectivamente) y no significativa en la tercera $(\rho=0,22 ; P=0,43)$.

La interacción EXP*EF no fue significativa $(\mathrm{P}$ $=0,15)$. Sin embargo, los cambios en la magnitud de $\Delta H^{\prime}$ 't por EF, t y EXP sugiere que este resultado debe ser revisado. La media de $\Delta H^{\prime}$ t para una misma EF a lo largo del tiempo es variable. En el segundo y tercer períodos, $\Delta \mathrm{H}^{\prime}$ t en algunas EF aumentó, en otras se mantuvo y/o disminuyó con respecto al período anterior, indicando en este último caso una caída de la diversidad por debajo de la inicial (Fig. 2B y 3B).

La hipótesis general de este trabajo se relaciona con los efectos del fuego sobre la diversidad herbácea, es decir efectos de segundo orden
(Reinhardt et al. 2001). Estos deben interpretarse teniendo en cuenta las características del fuego, el tamaño del área modificada (escala), la composición botánica del área afectada y la sensibilidad de las distintas especies al fuego (Wright \& Bailey 1982, Collins \& Barber 1985, Silva 1987, Howe 1995, 1999, Bravo \& Jiménez-Mosca 1996, Ford 1999).

Los fuegos obtenidos en este estudio fueron de intensidad y severidad similares a los que ocurren habitualmente en las sabanas de la región chaqueña (Bravo et al. 2001). Los resultados generales de este experimento indican un efecto positivo de esos fuegos sobre la diversidad de herbáceas del aibal; similar al comprobado en otros ecosistemas de pastizales y sabanas de la Argentina y el mundo (Wright \& Bailey 1982, Trollope 1984, Hulbert 1988, Nuñez et al. 2001).

Cambios en los componentes de la diversidad implican cambios en la presencia de especies y/o en la magnitud del reclutamiento de individuos. Una observación de los datos de campo indica que luego del fuego, especies poco frecuentes incrementaron su población y el tamaño de las plantas. Son ejemplos de ellos Zexmenia spp., 
(A)

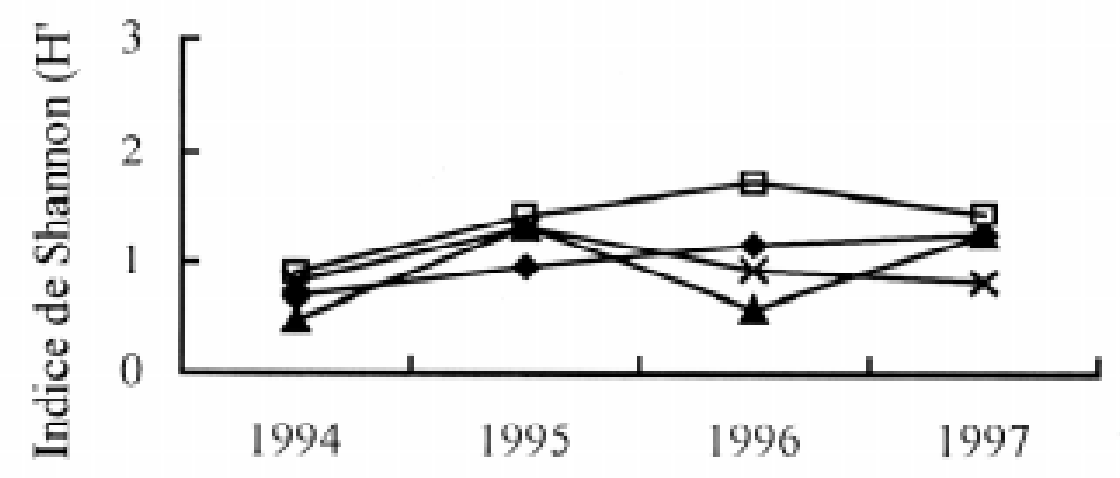

Año de observación

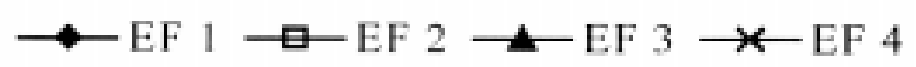

(B)

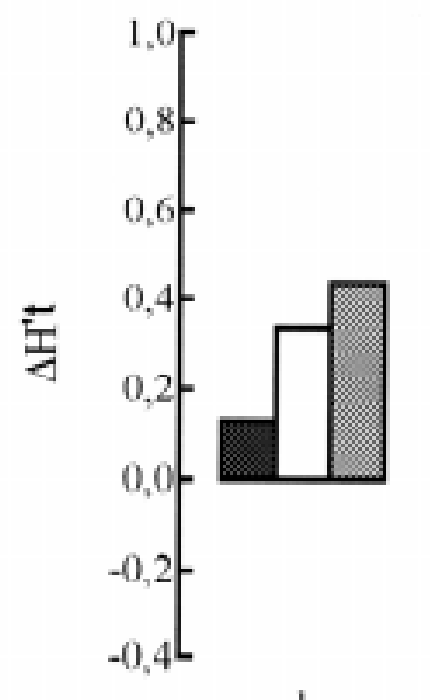

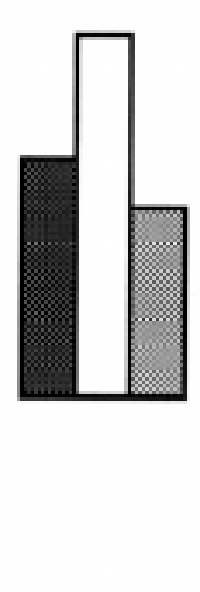

2

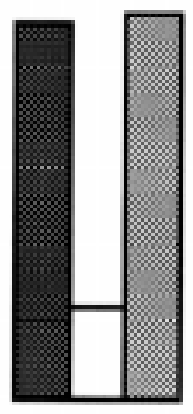

3

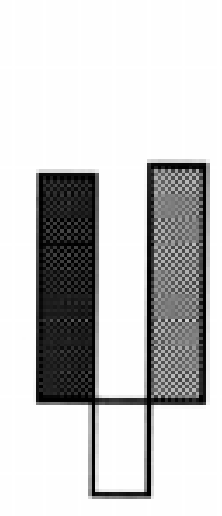

4

Fecha de aplicación de fuego

붐 1995-1994 ㅁ 1996-1994 뭄 1997-1994

Fig. 3: Dinámica a través del tiempo de: (A) índice de diversidad de Shannon-Wiener (H'); y (B) diferencia entre $H^{\prime}$ observados antes y despúes del fuego $\left(\Delta H^{\prime} t\right)$ para cuatro épocas de quema de una sabana de Elionorus muticus (Spreng) O. Kuntze . Ensayo iniciado en 1994 (EXP 2). Campo Experimental 'La María', EEA Santiago del Estero. EF: Epocas de fuego. Otros datos en Tabla 1 y en el texto.

Temporal dynamics of: (A) Shannon-Wiener diversity index (H'); and (B) difference of H' observed before and after the fire $\left(\Delta H^{\prime} t\right)$, in four fire application dates in a Elionorus muticus (Spreng.) O. Kuntze savanna. Experiment initiated in 1994 (EXP 2). 'La María' Experimental Ranch, INTA EEA Sgo del Estero. EF: Fire application dates. For other information see Table 1 and text. 
Indigofera parodiana, Baccharis spp. y Adesmia muricata. En el EXP 2 las plántulas de gramíneas influyeron positivamente sobre H', observándose germinación de Heteropogon contortus y Pappophorum pappipherum. No se observó germinación de individuos de aibe, la especie dominante, aunque las plantas florecieron abundantemente.

Los cambios en el número y/o tamaño de individuos se atribuyen a que el fuego produce mayor disponibilidad de luz, remoción del mantillo, aumento de: (a) temperatura del suelo, (b) del reciclaje de nutrientes; y (c) de la actividad biológica edáfica. Estos cambios afectan la germinación de las semillas y la actividad fisiológica de las plantas (Wright \& Bayley 1982).

La significancia del término EF en el corto plazo y fase inicial del largo plazo indican además que estos efectos generales del fuego pueden variar de acuerdo con las circunstancias climáticas y biológicas asociadas al momento de la quema modificando el impacto del mismo sobre la diversidad herbácea. En el corto plazo, los cambios nulos o negativos en la diversidad en fechas tempranas (EF 1) se atribuyen al largo período de tiempo durante el cual el suelo y las plantas quedaron expuestos a las condiciones climáticas desfavorables que se vieron reflejadas en la mortalidad de individuos adultos y jóvenes, esta última a causa de la germinación temprana de semillas. Por el contrario, quemas tardías (EF 3 y 4) tuvieron efectos positivos sobre la diversidad, que aumentó de manera significativa con respecto a la observada antes del fuego. El comienzo de la estación de lluvias es un factor que contribuye también a explicar esta diferencia de efectos de la fecha de aplicación de fuego.

En el largo plazo, el efecto del fuego también es diferente para cada fecha de aplicación, observándose una fuerte influencia de la diversidad inicial, representada en el análisis por las diferencias entre sitios experimentales y expresada en la interacción $\left(\mathrm{EF}^{*} \mathrm{EXP}\right)$. Las fechas de aplicación de fuego de media estación (EF 2 y EF 3) son las que presentaron mayores incrementos en diversidad con respecto a las tempranas (EF 1) y tardías (EF 4). Este efecto se atribuye a que las condiciones climáticas postquema son mejores que en las quemas tempranas; y a que las plantas se encontraban en condiciones de receso vegetativo, durante el cual su metabolismo está ajustado para soportar más exitosamente condiciones de estrés (Larcher 1995).

La disminución de la magnitud media de $\Delta \mathrm{H}^{\prime}$ t a través del tiempo y la ausencia de correlación entre la $\mathrm{H}^{\prime}$ inicial y $\mathrm{H}^{\prime}$ observada en el tercer $\mathrm{t}$ luego del fuego demuestran que el efecto del fuego como perturbación sobre la diversidad se 'diluye' en el tiempo. Así, después de tres estaciones de crecimiento postfuego los cambios de diversidad respecto a la diversidad inicial son nulos. El patrón indefinido en el comportamiento de $\Delta H^{\prime}$ t luego de la segunda estación de crecimiento puede atribuirse también a que otros factores tales como la disponibilidad de luz y la competencia entre especies ejercen mayor influencia sobre la diversidad. Así, por ejemplo, la desaparición de especies anuales, bianuales y/o perennes de tamaño reducido sería producto del aumento de la cobertura del aibe y la consiguiente reducción de la disponibilidad lumínica. La acumulación de mantillo también disminuye la disponibilidad de luz para germinación (Wright \& Bailey 1982).

La significancia de la interacción EXP*EF pone en debate la repetibilidad del efecto de EF sobre la diversidad. Este efecto se atribuye al comportamiento de la media de $\Delta \mathrm{H}^{\prime} \mathrm{t}$ en los períodos $\mathrm{t}=$ $2 \mathrm{y} \mathrm{t}=3$, cuando los efectos del fuego sobre la diversidad se 'diluyen'. En el corto plazo y en la fase inicial del largo plazo, el efecto del fuego es positivo en todos los casos, difiriendo sólo en su magnitud.

El efecto positivo general del fuego sobre la diversidad de herbáceas de la sabana a corto plazo y la desaparición del mismo en 1-3 años coinciden con estudios similares llevados a cabo en pastizales templados (Collins \& Barber 1985, Howe 1995, 1999) y en zonas serranas áridas (Núñez et al. 2001).

\section{CONCLUSIONES}

El fuego como perturbación crea un parche de tamaño variable en las sabanas y es un elemento clave en la dinámica de esta comunidad al crear espacio para la regeneración y crecimiento de especies anuales y perennes. Las parcelas empleadas en este trabajo son porciones pequeñas de una unidad de paisaje mayor, la sabana. Su tamaño es arbitrario, en función de la facilidad de manejo del fuego; y la extrapolación de los resultados obtenidos en las mismas para generar recomendaciones de manejo puede ser objeto de debate. Sin embargo, aún teniendo en cuenta la variabilidad de diversidad inicial, los resultados obtenidos apoyan la hipótesis que el fuego, en términos generales, es un pulso necesario para mantener la diversidad herbácea de los aibales de la región chaqueña. Su efecto, además de controlar leñosas e impedir la 'arbustificación' sería limitar la dominancia (cobertura) de aibe y facilitar la presencia de otras especies. 
A partir de los resultados obtenidos en este trabajo podemos inferir que el fuego aumenta la diversidad de especies en las sabanas chaqueñas en el período de 6 meses hasta 2 años de ocurrido. La recurrencia del fuego a lo largo del tiempo con intervalos libres de incendios de 2 a 3 años contribuirían a mantener la diversidad herbácea de los aibales, manteniendo la sabana como hábitat. Desde un punto de vista de manejo de la sabana, este estudio sugiere que la aplicación de quemas con objetivos de conservación de especies herbáceas deberían ser implementadas a mediados de la temporada de fuego.

\section{AGRADECIMIENTOS}

Los autores agradecen al personal del Campo Experimental 'La María' la colaboración prestada en la implementación de fuegos. Este trabajo fue financiado por el Instituto Nacional de Tecnología Agropecuaria, Centro Regional TucumánSantiago del Estero, a través del Plan de Trabajo No. 420117, "Ecología y manejo de fuego en la región chaqueña semiárida".

\section{LITERATURA CITADA}

ALEXANDER M (1982) Calculating and interpreting forest fire intensities. Canadian Journal of Botany 60: 349357.

ANDERSON DL (1980) Manejo racional de un campo en Los Llanos de La Rioja (República Argentina). Instituto Nacional de Tecnología Agropecuaria, Buenos Aires, Argentina. 90 pp.

BEGON M, H HARPER \& M TOWNSEND (1997) Ecología: individuos, poblaciones y comunidades. Editorial Omega, Barcelona, España. 300 pp.

BOLETTA P (1988) Clima. En: Casas R (ed) Desmonte y habilitación de tierras en la región chaqueña semiárida: 7-21. FAO, Santiago, Chile.

BONHAM C (1989) Measurements for terrestrial vegetation. Wiley-InterScience, John Wiley \& Sons, New York. New York. x +338 pp.

BORDÓN A (1993) Notas sobre incendios en la región chaqueña con base en una encuesta para información sintética sobre incendios de campos. En: Kunst C, A Sipowicz, N Maceira \& S Bravo (eds) Ecología y manejo de fuego en ecosistemas naturales y modificados: 69-74. Instituto Nacional de Tecnología Agropecuaria, Estación Experimental Agropecuaria Santiago del Estero, Argentina.

BRAVO S \& A JIMÉNEZ-MOSCA (1996) Efectos del fuego sobre las plantas. En: Kunst C \& F Moscovich (eds) Introducción a la ecología de fuego y manejo de fuego prescripto: 29-36. Instituto Nacional de Tecnología Agropecuaria, Estación Experimental Agropecuaria Santiago del Estero, Argentina.

BRAVO S, C KUNST, A JIMÉNEZ \& G MOGLIA (2001) Fire regime of a Elionorus muticus Spreg. savanna, western Chaco region, Argentina. International Journal of Wildland Fire 10: 1-8.

BRITTON C, H WRIGHT, B DAHL \& D UECKERT (1987) Management of tobosagrass rangeland with prescribed fire. Management Note 12, Department of Range and Wildlife, Texas Tech University, Lubbock, Texas. 3 pp.

BUCHER E (1982) Chaco and Caatinga: South American arid savannas, woodlands and thickets. En: Huntley B \& B Walker (eds) Ecology of tropical savannas: 4879. Springer-Verlag, Berlín, Germany.

BURKART G (1969) Flora ilustrada de Entre Ríos. Instituto Nacional de Tecnología Agropecuaria, Colección Científica, Tomo VI, Parte 2, Buenos Aires, Argentina. Xv + 551pp.

BURKE I, W LAUENROTH, R RIGGLE, P BRANNEN, B MADIGAN \& S BEARD (1999) Spatial variability of soil properties in the shortgrass steppe: the importance of topography, grazing. microsite and plant species in controlling spatial patterns. Ecosystems 2: 422-438.

CABRERA A \& A WILLINK (1973) Biogeografía de América Latina. Organización de Estados Americanos, Serie de Biología, Monografía 13, Washington, District of Columbia. $130 \mathrm{pp}$.

CARR M (1996) PRIMER user's manual (Plymouth routines in multivariate ecological research). Plymouth Marine Laboratories, Plymouth, United Kingdom. 20 pp.

CLARKE K \& R WARWICK (1994) Change in marine communities: an approach to statistical analysis and interpretation. Natural Environment Research Council, Plymouth, United Kingdom. 150 pp.

COLLINS S \& S BARBER (1985) Effects of disturbance on diversity in mixed prairie. Vegetatio 64: 87-94.

CONOVER WJ (1980) Practical nonparametric statistics. John Wiley \& Sons, New York, New York. xiv +493 pp.

COX D (1958) Planning of experiments. John Wiley \& Sons, New York, New York. 308 pp.

DIXON W (ed) (1992) BMDP statistical software, release 7, Volume 2. University of California, Berkeley, California. $\mathrm{xx}+678 \mathrm{pp}$.

FORD P (1999) Response of buffalograss (Buchlö̈ dactyloides) and blue grama (Bouteloua gracilis) to fire. Great Plains Research 9: 261-76.

FREUND R, R LITTELL \& P SPECTOR (1986) SAS system for linear models. SAS Institute Inc., Cary, North Carolina. 150 pp.

GILL A M \& M MCCARTHY (1998) Intervals between prescribed fires in Australia: what intrinsic variation should apply? Biological Conservation 85: 161-169.

HANSMIRE J, D LYNN DRAWE, D WESTER \& C BRITTON (1988) Effect of winter burns on forbs and grasses of the Texas coastal prairie. Southwestern Naturalist 33: 333-338.

HOWE H (1994) Managing species diversity in tallgrass prairie: assumptions and implications. Conservation Biology 8: 691-704.

HOWE H (1995) Succession and fire season in experimental prairie plantings. Ecology 76: 1917-1925.

HOWE H (1999) Dominance, diversity and grazing in tallgrass restoration. Ecological Restoration 17: 5966. 
HULBERT L (1988) Causes of fire effects in tallgrass prairie. Ecology 69: 46-58.

JORGENSEN S \& J PADISAK (1996) Does the intermediate disturbance hypothesis comply with thermodynamics? Hydrobiologia 323: 9-21.

KIRKAM LK, M DREW \& D EDWARDS (1998) Effects of experimental fires on the population dynamics of Schwalbea americana L. Plant Ecology 137: 115137.

KUNST C, H PÉREZ, M PEROTTI, R GIORGIS \& J APESTEGUI (1998) Intensificación de la producción ganadera en el sudeste de la Provincia de Santiago del Estero: II. Análisis de la oferta de forraje del campo natural. Memorias del Primer Congreso Nacional Cambio Rural: 64-65. Secretaría de Agricultura, Ganadería, Pesca y Alimentación, Buenos Aires, Argentina.

KUNST C, R RENOLFI, H PÉREZ, A SMERIGLIO, N SUEIRO \& F DALLATEA (1985) Elionorus muticus Spreng. Información Técnica, Área Recursos Naturales y Producción Animal No. 7. Instituto Nacional de Tecnología Agropecuaria, Estación Experimental Agropecuaria Santiago del Estero, Argentina. 4 pp.

KUNST C, S BRAVO, F MOSCOVICH, J HERRERA, J GODOY \& S VELEZ (2000) Control de tusca (Acacia aroma Gill ap. H. et A.) mediante fuego prescripto. Revista Argentina de Producción Animal 20: 199213.

LARCHER W (1995) Physiological plant ecology. Springer, Berlín, Germany. 330 pp.

LITTELL R, P HENRY \& C AMMERMAN (1998) Statistical analysis of repeated measures data using SAS procedures. Journal of Animal Science 76:12161231.

LUDWIG J \& J REYNOLDS (1988) Statistical ecology. John Wiley \& Sons, New York, New York. xvii +337 pp.

MORELLO J \& C SARAVIA-TOLEDO (1959) El bosque chaqueño I y II. Revista Agronómica del Noroeste Argentino 3: 5-81/209-258.

MORELLO J \& J ADÄMOLI (1974) Las grandes unidades de vegetación y ambiente del Chaco argentino. Segunda Parte. Instituto Nacional de Tecnología Agropecuaria, Serie Fitogeográfica No. 13, Buenos Aires, Argentina. $130 \mathrm{pp}$.

NÚÑEZ CO, JJ CANTERO, M REYNERO, J CISNEROS \& A DEGIOANNI (2001) Cambios estructurales en pastizales naturales afectados por fuego y pastoreo en la Sierra de Comechingones, Córdoba (Argentina). Congreso para la Prevención y combate de incendios forestales y de pastizales del MERCOSUR, Asocia- ción de Amigos de Parques Nacionales, Villa Carlos Paz, Córdoba, Argentina. 250 pp.

ORR D, C. PATON \& T LISLE (1997) Using fire to manage species composition in Hetoropogon contortus (black speargrass) pastures. Australian Journal of Agricultural Research 48: 795-802.

REINHARDT E, R KEANE \& J BROWN (2001) Modelling fire effects. International Journal of Wildland Fire 10: $373-380$.

RENOLFI R (1993) Dinámica de una sabana de Elionorus muticus Spreng. (aibe) sometida a fuegos fortuitos. En: Kunst C, A Sipowicz, N Maceira \& S Bravo (eds) Ecología y manejo de fuego en ecosistemas naturales y modificados: 171-176. Instituto Nacional de Tecnología Agropecuaria, Estación Experimental Agropecuaria Santiago del Estero, Argentina.

ROTHERMEL R (1983) How to predict the spread and intensity of forest and range fires. United States Forest Service, Intermountain Forest and Range Experimental Station, Missoula, Montana, GTR INT-143. 150 pp.

RYAN K \& N NOSTE (1985) Evaluating prescribed fires. En: Lotan J, B Kilgore, W Fischer \& R Mutch (eds) Symposium and workshop on wilderness fire: 230 238. United States Forest Service, Intermountain Forest and Range Experimental Station, Missoula, Montana, GTR INT-182. 300 pp.

SAS INSTITUTE INCORPORATED (1990) SAS/ STAT user's guide, version 6, Volume 1. Fourth Edition. Cary, North Carolina. 600 pp.

SILVA JF (1987) Responses of savannas to stress and disturbances: species dynamics. En: Walker BH (ed) Determinants of tropical savannas 141-156. International Union of Biological Sciences, Monograph Series 3, Paris, France.

TÁLAMO A (1999) Diversidad de plantas leñosas y disturbios antrópicos en el bosque subtropical semiárido. Tesis Profesional, Facultad de Ciencias Naturales, Universidad Nacional de Salta, Salta, Argentina. ii + $230 \mathrm{pp}$.

TOTHILL J (1969) Soil temperatures and seed burial in relation to the performance of Heteropogon contortus and Themeda australis in burnt native woodland pastures in eastern Queensland. Australian Journal of Botany 17: 269-275.

TROLLOPE WSW (1984) Fire in savanna. En: Booysen PV \& N Tainton (eds) Ecological effects of fire in South African ecosystems: 149-197. Springer-Verlag, Berlin, Germany.

UNDERWOOD A (1998) Experiments in ecology. Cambridge University Press, Cambridge, United Kingdom. xviii + 504 pp.

WRIGHT H \& H BAILEY (1982) Fire ecology in the United States and Canada. John Wiley \& Sons, New York, New York. xxi + 501 pp.

Editor Asociado: Julio Gutiérrez

Recibido el 14 de diciembre de 2001; aceptado el 27 de junio de 2002 